\title{
XLVI. On the theory of pyro-electricity and piezo- electricity of crystals
}

\section{Lord Kelvin}

To cite this article: Lord Kelvin (1893) XLVI. On the theory of pyro-electricity and piezo-electricity of crystals, Philosophical Magazine Series 5, 36:222, 453-459, DOI: 10.1080/14786449308620501

To link to this article: http://dx.doi.org/10.1080/14786449308620501

曲 Published online: 08 May 2009.

Submit your article to this journal $\sqsubset \pi$

Џ Article views: 7

Q View related articles $\sqsubset$ 
geneous mass must have presented themselves in a very striking manner at higher temperatures and under stronger pressures, and at a certain stage resulted in the production of the above optically homogeneous appearance.

It is therefore very likely, as Jamin remarked, that we have under those circumstances of temperature and pressure nothing that is different in kind from what we see under ordinary circumstances.

Dewar's experiments, especially those on liquefaction of carbonic acid in presence of other liquids, are interesting from our point of view, and we cannot but think of some such process as interdiffusion being in action through all cases, not excepting the case of pure gases.

XLVI. On the Theory of Pyro-electricity and Piezoelectricity of Crystals. By Lord Kelvis*.

$\S 1.7 \mathrm{HE}$ doctrine of bodily electro-polarization masked by an induced superficial electrification, which I gave thirty years ago in Nichol's Cyclopacdia $\dagger$, wanted a physical explanation of the assumed molecular polarization to render it a satisfying physical theory of pyro-electricity; and it was essentially defective, as has been remarked by Röntgen $\ddagger$ and by Voigt $\S$, in that it contained no suggestion towards explaining the multiple electric polarities irregularly produced by irregular changes of temperature in boracite, in quartz, and in tourmaline itself; which had perplexed many naturalists and experimenters. A short but very important paper by MM. Jacques and Pierre Curie in the Comptes Rendus for Feb. 14, 1881, supplies that want in a manner which suggests what seems to me the true matter-of-fact electro-chemical theory of a crystalline molecule, and at the same time makes easy the extension of my slight primitive doctrine, to remedy its defect in respect to multipolarity, and to render it available for explaining not only the old-known py ro-electricity of crystals, but also the piezo-electricity discovered by the brothers Curie $\|$ themselves. The element of zinc and copper soldered together and surrounded only by air, which they suggest, represents perfectly a true electro-

* Communicated by the Author.

+ Reprinted in 'Collected Mathematical and Physical Papers' (Sir W. Thomson), vol. i. p. 315 .

† Wiedemann's Annalen, 1883, xviii. p. 213.

$\$$ "Allgemeine Theorie der Pjëzo- und Pyro-electrischen Erscheinungen an Krystallen," p. 6 ; separate publication from vol. xxxvi. of Abhand. König. Ges. Wiss. Göttingen, 1890.

If Comptes Rendus, Aug. 2 and Aug. 16, 1880. 
chemical compound molecule such as $\mathrm{H}_{2} \mathrm{O}$ or $\mathrm{SiO}_{2}$ in a realizable model, which indeed $I$ actually made three weeks ago and described in a communication to the Philosophical Magazine * without knowing that I had been anticipated.

\$2. To represent pyro-electric and piezo-electric qualities in a crystal, take as crystalline molecule a rigid body of any shape, bounded by a surface made up of pieces of different metals, soldered together so as to constitute one metallic conductor. Arrange a large number of such molecules in order, as a Bravais homogeneous assemblage, not touching one another. Connect every molecule with neighbours by springs of non-conducting material (india-rubber may be taken if we wish to make a practically working model). We may, for example, suppose each molecule to be connected with only twelve neighbours; its two nearest, its two next-nearests, its two next-next-nearests in the plane of those four, and the three pairs of nearests, next-nearests, and next-next-nearests on the two sides of that plane. Thus we have a perfect mechanical model for the elasticity and the piezo-electricity of a crystal; and for pyro-electricity also, if we suppose change of temperature to produce either change of the contact-electricities of the metals, or change of configuration of the assemblage, whether by changing the shape of each molecule or by changing the forces of the springs.

$\S 3$. The mathematical problem which this combination presents is as follows:-

Given a homogeneous assemblage of a large number of equal and similar closed surfaces, $S$, each composed of two or more different kinds of metal soldered together, all insulated in a large closed chamber, of which the bounding surface $\mathrm{C}$ is everywhere at a practically infinite distance from the assemblage, and is of the same metal as one of the metals of $\mathrm{S}$, copper we shall suppose, to fix the ideas.

It is required to find :-

(1) The potential in the copper of every molecule when the total quantity of electricity on each is zero.

(2) The quantity of electricity on each molecule when all are metallically connected by infinitely fine wire.

$\S 4$. The mathematical expression of the conditions and requirements of the problem is as follows :-

Let $f(\mathrm{P})$ denote a given function of the position of a point $P$ on the surface $S$ of any one of the molecules; expressing the difference of the potential in the air infinitely near to $P$, from the potential in the air infinitely neur to the copper

* For October 1893, " On a Piezo-electric Pile." 
parts of the surface S. This function is the same for corresponding points of all the molecules.

Let $\mathrm{V}_{n}$ be the potential at the copper of the molecule numbered $n$.

Let $\mathrm{D}\left(\mathrm{P}_{i}, \mathrm{P}_{n}\right)$ be the distance between a point $\mathrm{P}_{i}$ on the molecule numbered $i$, and a point $\mathrm{P}_{n}$ on the molecule numbered $n$.

Let $\iint d s_{i}$ denote integration over the surface of molecule $i$, and $\rho_{i}$ a function of the position of $P_{i}$ on the surtace of this molecule; (the electric density at $P_{i}$ ).

Let $\Sigma_{i}$ denote summation for all the molecules, including the case $i=n$.

Let $q_{n}$ be the total quantity of electricity on molecule $n$.

The equation of electric equilibrium is

and we have

$$
\Sigma_{i} \iint \frac{\rho_{i} d s_{i}}{\mathrm{D}\left(\mathrm{P}_{i}, \mathrm{P}_{n}\right)}=f\left(\mathrm{P}_{n}\right)+V_{n} \quad . \quad . \quad(a),
$$

It is required to find

$$
\iint \rho_{n} d s_{n}=q_{n} \quad . \quad . \quad . \quad . \quad . \quad . \quad(b) .
$$

(1) $\mathrm{V}_{n}$, when $q_{n}=0$, for every value of $n$;

and (2) $q_{n}$, when $\mathrm{V}_{n}=0, \quad, \quad$,

$\S 5$. The problem thus proposed is of a highly transcendental character, unless the surface $S$ is spherical. In this case it can be solved for any finite number of molecules by mere expenditure of labour ; perhaps the work of the natural working-life of a competent mathematician, if the assemblage is a Bravais parallelepiped of 125 globes in 5 " réseaux" of 25 globes each, would suffice to give the solution for each item within one per cent. of accuracy; and not much more labour would be needed to solve the problem to the same degree of accuracy for each of $125 \times 10^{21}$ spherical molecules in a similar Bravais parallelepiped of $5 \times 10^{7}$ "réseaux," if the distance ( $h$ we shall call it) between the planes of corresponding points of two consecutive "réseaux" of nearest and next-nearest molecules is not greater than about twice the diameter of each molecule.

$\S 6$. When this last condition is fulfilled, we can see, from general knowledge of the doctrine of electric screening, without solving the problem as proposed for every individual molecule, that the solution of the second part (2) of the requirements is $g_{n} \fallingdotseq 0$, very approximately for every molecule at any distance exceeding two or three times $h$ from every 
part of the boundary of the assemblage ; and this whether the molecules are spherical, or of any other shape not too wildly different. We see also that for all molecules not nearer than $3 \dot{h}$, or perbaps $4 h$, or $5 h$, from any part of the boundary of the assemblage, the distribution of electricity is similar. That is to say, the whole assemblage within a thin surface-layer (of some such thickness as $3 h$ or $4 h$ ) is homogeneous, not only geometrically and mechanically, but also electrically. The problem of finding, with moderate accuracy, the distribution of electricity on each molecule of the homogeneous assemblage thus constituted, is comparatively un-laborious if the shape of each molecule is spherical.

$\S 7$. We also see, by the known elements of electrostatics, without solving the problem of finding the quantity of electricity on each molecule of the surface-layer in the circumstances described in $\S 6$, that the sum of the quantities on all the molecules of this layer, per unit of the surface, is equal to the component, in the direction normal to the surface, of the electric moment per unit-volume of the homogeneous assems blage within the surface-layer.

\$ 8 . The condition of the whole assemblage, surface-layer and homogeneous assemblage within it, at which we have arrived in $\$ \S 6$ and 7 , may be regarded as representing the natural undisturbed condition of a crystal. Let now any homogeneous change of configuration of our assemblage be produced either by proper application of force to the molecules of the surface-layer, or by uniform change of temperature throughout the interior, or by both these causes acting simultaneously. We need not exclude the case of no change of shape or bulk of the boundary ; that is to say, the case of no change of the relative positions of corresponding points of the molecules; and our "change of configuration" only an infinitesimal rotation of each molecule. The inclusion of this case is important to guard against a tendency which I find in the writings both of MM. Curie and of Voigt :- a tendency to a hypothetical assumption unduly limiting the pyro-electric property to identity with the piezo-electric effect produced by force causing the same change of shape or bulk as that which is produced by the change of temperature. In nature, we may expect as a general possibility, and as a probable result in some cases, a bodily electro-polarization produced by change of temperature, even though change of bulk and shape are prevented by force applied to the surface. And, in our model, changes of forces of the springs would certainly cause rotation of the molecules, and so produce electro-polarization, even when the molecules of the boundary are held fixed, unless the 
springs are specially designed and constructed to annul this effect.

$\S 9$. Solve now the electrical problem of finding the change of electric moment of each molecule of the homogeneous assemblage, produced by the change of configuration described in $\S 8$, when the potential is zero throughout the surface-layer. To avoid tampering with the separate insulation of all or any of the molecules, and to conform our ideas to the realities of experiments on the electric properties of crystals, I suppose this equality of potential to be produced not by temporary metallic connexion between the molecules as in $\S 3(2)$, but by a metal coat enclosing our model, and having its inner surface everywhere very near to the boundary of the assemblage; for example, everywhere within a distance of less than $2 h$ or $3 h$ in our model, or of less than $10^{3} \times h$ if we are dealing with a real crystal in a real experiment.

$\$ 10$. To find experimentally the solution of the mathematical problem of $\$ 9$, divide the metal coat into two parts ; one of them (corresponding to Coulomb's "proof-plane") we shall call for brevity $\mathrm{E}$. It may be either so small that it is sensibly plane, or it may be a portion of the coat covering a finite plane part of the boundary of the assemblage. Commence now with the crystal in its natural undisturbed state, and having the metal coat on it with $\mathrm{E}$ insulated from the rest of the coat. Produce a change of configuration as in $\$ 8$; and then measure how much electricity would need to pass from $\mathrm{E}$ to the rest of the coat to equalize the potential between them. This is wholly and exactly what MM. Curie do in their admirably designed measurement with their "quartz piézo-électrique," avoiding all need for consideration of the essentially transcendent problem of the distribution of electric potential at the surface of an uncoated crystal when there is either pyro-electric or piezo-electric disturbance of its interior.

The quantity of electricity thus measured, divided by the area of $\mathrm{E}$, is equal to the component perpendicular to $\mathrm{E}$ of the interior electro-polarization when $\mathrm{E}$ and the rest of the coat are metallically connected.

$\S 11$. In conclusion, following Voigt in his Allgemeine Theorie, already referred to, we see that there are essentially 18 independent coefficients for the piezo-electricity of a crystal in general; in three formulas expressing the three components of the electric moment per unit of its volume each as a linear function of the six components of the geometrical strain of the substance. To each of these expressions I add a term for the component of the electric moment due 
to change of temperature when force acting on the surface prevents change of volume or shape. Thus we have in all 21 independent coefficients for piezo-electricity and pyroelectricity; to be determined for a real crystal by observation. It is interesting to see how our model can be constructed to realize the piezo-electric and pyro-electric phenomena in accordance with any given values of these 21 coefficients, by experimental solution of as much of the mathematical problem of $\& 4$ as is necessary for the purpose.

$\$ 12$. Choose any convenient shape, spherical or not wildly different from spherical, for each molecule. Divide the whole surface into 22 parts (not wildly unequal nor extravagantly different from squares or equilateral equi-angular hexagons), and number them $0,1,2, \ldots 21$. Construct a trial molecule with part 0 always of copper; and with, for first trial, part 1 of zinc, and parts $2,3, \ldots 21$ of copper. Take a large number of such molecules and make of them a Bravais homogeneous assemblage with any arbitrarily chosen values for the six edges of the fundamental acute-angled tetrahedrons. Connect the molecules homogeneously by springs of non-conducting material in the manner described in $\$ 2$ above. To provide fully for pyro-electricity, withont hypothesis, we must now take care that these springs are such, that when the temperature is changed, and the border molecules are held fixed, all the interior molecules shall be caused to rotate round parallel axes through equal angles proportional to the difference of temperature. For this purpose the springs must be of two or more different materials; and when set in their proper positions between the molecules they must be under stress, some of them pushing and some pulling, in the undisturbed condition of the assemblage.

$\S 13$. Subject now the assemblage successively to six different geometrical strain-components, $e, f, g, a, b, c$; and to one change of temperature, $t$, with the boundary molecules held fixed. With each of these seven configurations, measure, by three separate measurements conducted according to the method described in $\S 10$, the three components of the sum of the electric moments of the molecules in unit volume.

$\S 14$. Repeat the same 21 measurements with part 2 of the surface of each molecule zinc, and all the rest copper : next with part 3 zinc, and all the rest copper : and so on. Thus we have $21^{2}$ distinct measurements, each giving independently one of the $21^{2}$ multipliers $[x, e, 1],[x, e, 2]$, \&c., which appear in the following 21 equations :- 


$$
\begin{aligned}
& {[x, e, 1] v_{1}+[x, e, 2] v_{2}+\ldots[x, e, 21] v_{21}=(x, e),} \\
& {[x, f, 1] v_{1}+[x, f, 2] v_{2}+\ldots[x, f, 21] v_{21}=(x, f),} \\
& {[x, g, 1] v_{1}+[x, g, 2] v_{2}+\ldots[x, g, 21] v_{21}=(x, g),} \\
& {[x, a, 1] v_{1}+[x, a, 2] v_{2}+\ldots[x, a, 21] v_{21}=(x, a),} \\
& {[x, b, 1] v_{1}+[x, b, 2] v_{2}+\ldots[x, b, 21] v_{21}=(x, b),} \\
& {[x, c, 1] v_{1}+[x, c, 2] v_{2}+\ldots[x, c, 21] v_{21}=(x, c),} \\
& {[x, t, 1] v_{1}+[x, t, 2] v_{2}+\ldots[x, t, 21] v_{21}=(x, t),}
\end{aligned}
$$

\&c., \&c., \&c., with $y$ and $z$ instead of $x$.

In these equations $v_{1}, v_{1}, \ldots v_{21}$ denote the volta-electric differences from copper which must be given to part 1, part $2, \ldots$ part 21 of the surface of the molecule in order that the 21 piezo-electric and pyro-electric coefficients may have their given values $(x, e),(x, f) \ldots(z, t)$; the meaning of these coefficients being explained by the following three equations:-

$\mathrm{X}=(x, e) e+(x, f) f+(x, g) g+(x, a) a+(x, b) b+(x, c) c+(x, t) t$, $\mathrm{Y}=(y, e) e+$. . . . . . . . . . . $+(y, t) t$, $\mathrm{Z}=(z, e) e+$. . . . . . . . . . . . $+(z, t) t$,

where $\mathrm{X}, \mathrm{Y}, \mathrm{Z}$ denote the components of the electric moment per unit of volume, produced in the erystal by geometrical change, and change of temperature $(e, f, g, a, b, c, t)$. Thus, the volta-electric difference of zinc from copper being taken as unity, the 21 volta-differences from copper, of parts 1 to 21 of the surface of each molecule, are determined by 21 linear equations.

$\S 15$. Thus we have, in idea, constructed a model for the piezo-electric and pyro-electric quality of a crystal in which each one of the 21 piezo-electric and pyro-electric coefficients has an arbitrarily given value.

XLVII. A Modifed Form of Bunsen and Roscoe's Pendulum Actinometer. By Dr. A. Richardson and Mr. J. Quick *.

TN Bunsen and Roscoe's pendulum actinometer (Proc. Royal Soc. 1862, p. 139), the time is measured in which a given intensity of light will produce a degree of darkening on sensitized paper equal in shade to a standard tint.

Without going into details, the apparatus may be briefly described as follows :-The oscillations of a pendulum cause an attached sliding shutter to pass backwards and forwards over a slit, in the direction of its longer axis, beneath which

* Communicated by the Authors. 